\title{
Normative beliefs and values that shape care-seeking behaviours for skilled birth attendance (SBA) during birthing by mothers in Africa: a scoping review protocol
}

\author{
Choolwe Jacobs ${ }^{1,2^{*}}$ (D) and Adnan A. Hyder ${ }^{3}$
}

\begin{abstract}
Background: Skilled birth attendance (SBA) during delivery has been associated with improved maternal health outcome. However, low utilisation of SBA during childbirth has continued in many developing countries including Zambia. The proposed scoping review aims to map literature on beliefs and values and how mothers are influenced by relational normative motivations in utilisation of SBAs in health facilities.

Methods: We designed and registered a study protocol for a scoping review. Literature searches will be conducted in multiple electronic databases (from January 2000 onwards), including PubMed, EMBASE, Scopus and Web of Science. Grey literature will be identified through searching dissertation databases, Google Scholar, EBSCOhost and ResearchGate. Keyword searches will be used to identify articles. Only articles published in English, related on beliefs and values surrounding childbirth, and on perceptions towards facility delivery or skilled health care providers will be considered eligible for inclusion. Two reviewers will independently screen eligible titles, abstracts and full articles with a third reviewer to help resolve any disputes. The study methodological quality (or bias) will be appraised using the Mixed Method Appraisal Tool. A narrative summary of findings will be conducted. We will employ NVIVO version 10 software package to extract the relevant outcomes from the included articles using content thematic analysis. This protocol is registered with the Open Science Framework (osf.io/9gn76).

Discussion: Understanding how individual mother's health seeking behaviours for SBA and those close to them are influenced by their beliefs and values is critical to informing health systems on the possible 'hidden' barriers and facilitators to utilisation of SBA in public health facilities. The review will complement evidence base on normative beliefs and values shaping care-seeking behaviours for skilled birth attendance by mothers in Africa.
\end{abstract}

Keywords: Skilled birth attendance, Beliefs, Values, Africa

\footnotetext{
* Correspondence: choolwe2003@yahoo.com

'School of Public Health, University of Zambia, Nationalist Road, Box 50110, Lusaka, Zambia

²Berman Institute of Bioethics, Johns Hopkins University, Baltimore, MD, USA

Full list of author information is available at the end of the article
}

(c) The Author(s). 2021 Open Access This article is licensed under a Creative Commons Attribution 4.0 International License, which permits use, sharing, adaptation, distribution and reproduction in any medium or format, as long as you give appropriate credit to the original author(s) and the source, provide a link to the Creative Commons licence, and indicate if changes were made. The images or other third party material in this article are included in the article's Creative Commons licence, unless indicated otherwise in a credit line to the material. If material is not included in the article's Creative Commons licence and your intended use is not permitted by statutory regulation or exceeds the permitted use, you will need to obtain permission directly from the copyright holder. To view a copy of this licence, visit http://creativecommons.org/licenses/by/4.0/ The Creative Commons Public Domain Dedication waiver (http://creativecommons.org/publicdomain/zero/1.0/) applies to the data made available in this article, unless otherwise stated in a credit line to the data. 


\section{Background}

Utilisation and access to quality facility-based services by skilled birth attendants (SBAs) during pregnancy, delivery, and post-delivery has been associated with improved maternal health outcomes $[1,2]$. However, low utilisation of care by SBAs during childbirth has continued in many developing countries.

Existing evidence reveals that individual and contextual factors, including health system factors, influence mothers' care-seeking behaviours for skilled birth attendance in health facilities during childbirth [3, 4]. Health system factors that hinder care seeking for childbirth have been reported to include limited availability of health care workers, lack of equipment and supplies $[5,6]$, and the attitude [7] and practices of health care workers, such as disrespectful and abusive care [8]. Individual level factors, such as the mother's age [9], marital status [10], level of education, economic status of the household [11, 12] and cultural and religious beliefs [13-16] have also been reported as negative predictors to care-seeking behaviours of mothers. The location of services available is another important contextual factor hindering utilisation of SBA for childbirth from the health facilities [17].

Some studies have reported that although some mothers are willing to seek care for childbirth [18] and would prefer to give birth in a health centre with a skilled health care provider [19], the perceptions of care provided by skilled attendants influences the intention and decision to seek care $[20,21]$. This becomes one of the contributing factors to the existing disparities to access and utilisation of care from SBAs during childbirth.

The growing need for health systems to ensure better maternal and infant health outcomes for populations requires continued exploration of possible hidden barriers using diverse approaches that inform policy. An understanding of the beliefs and values and how individual mothers are influenced by normative motivations is critical to informing public health policy on the possible 'hidden' barriers and facilitators to utilisation of SBA in public health facilities. Normative motivations are concerned with the individual's view of what is morally right and wrong [22]. These motivations involve doing the right thing just because it is right, and not as a means to avoid punishment or gain social rewards. Down and colleagues (2018) argue that the care of mothers during childbirth should be designed to fulfil women's personal and socio-cultural beliefs and expectations [23]. Therefore, studies focusing on mothers' normative values and beliefs and how these shape their decisions to seek care from a skilled birth attendant during childbirth need to be explored. However, the extent of the evidence on mothers' normative values and beliefs and how these values may be shaping their decisions and motivation to access care by a skilled birth attendant is limited. Therefore, this scoping review aims at mapping and documenting existing literature on normative beliefs and values that shape care-seeking behaviours for SBA among mothers in Africa. We hope that the findings from this review will highlight gaps in the existing literature and form a basis for refining research questions for future interventions.

\section{Methods}

\section{Search strategy}

A systematic search, selection, and synthesis of existing literature will be used through a scoping review methodology in order to answer the research question. Scoping reviews are a comprehensive and rigorous method aimed at rapidly mapping key concepts underpinning a research area. This review protocol has been registered within the Open Science Framework database (registration number: osf.io/9gn76) and is being reported in accordance with the reporting guidance provided in the Preferred Reporting Items for Systematic Reviews and Meta-Analyses Protocols (PRISMA-P) statement [24] (see checklist in Additional file 1). The scoping review methodology will be conducted in accordance with the framework proposed by Arksey and O'Malley [25], which has been further developed by Levac et al. [26].

\section{Identifying the research question}

Our research question is: What is the evidence of normative beliefs and values that shape care-seeking behaviours for SBA by mothers in Africa?

We have used the Population-Concept-Context (PCC) approach recommended by the Joanna Briggs Institute for scoping reviews [27] to construct a clear and meaningful research question for a scoping review.

\section{Identifying relevant studies}

The primary studies that have a clear empirical base utilising qualitative, quantitative and mixed methods published in peer-reviewed journals will be sourced based on a structured search of electronic databases (from January 2000 onwards): PubMed, EMBASE, Scopus and Web of Science. The secondary source of potentially relevant material will be a search of dissertation databases, Google Scholar, EBSCOhost and ResearchGate. Not only references of included documents but also key journals, thesis and dissertations will be hand-searched to identify any additional evidence sources. We will also search reference lists for relevant articles and studies. Review articles will be excluded. Medical Subject Headings $(\mathrm{MeSH})$ terms will be included during the search for relevant articles. The search strategy will be designed by a research librarian and peer reviewed. The search terms will include perception, expectation, experience, 
mothers, women, normative belief, culture, value, norm, tradition, utilization, utilisation, uptake, maternity, delivery, childbirth, sub-Sahara, Africa developing and lowand middle-income country. We will also use Boolean terms, AND and OR to separate the keywords. The search strategy will be adapted for each database. For each search conducted, we will document in detail the date of search, the search engine and the number of publications retrieved.

\section{Study selection}

Identification of appropriate and relevant articles will be guided by selection criteria.

\section{Eligibility criteria Inclusion criteria}

- Focus on women's beliefs and values surrounding childbirth

- Report on experiences and perceptions towards facility delivery or skilled health care providers

- Published from January 2000 onwards

- Studies conducted in Africa

\section{Exclusion criteria}

We will exclude the following articles:

- Studies with no evidence of mother's values, beliefs or norms surrounding childbirth

- Studies not focused on childbirth or delivery services

- Studies not freely available in full text

\section{Screening and selection process}

The search strategies will be piloted to check the appropriateness of the selected databases and key words. All the articles retrieved from the databases will be screened for eligibility through the following stages. In the first stage, the principle investigator (PI) will screen the all the titles for eligibility, and articles that meet the eligibility criteria will be exported to endnote, a referencing software (version 7) used to store and organize citation information. All the titles that are not eligible will be excluded and all the duplicates deleted. In the second stage, the PI and two independent trained reviewers will independently (in parallel) screen the abstracts from the retrieved titles to include only eligible articles. In the last stage, the PI and two independent reviewers shall read through the full articles for eligibility. At every stage, any differences in the outputs will be resolved through a discussion and a consensus will be derived. Reasons for exclusion of articles at each stage will be documented. A flow chart showing details of studies included and excluded at each stage of the study selection process will be provided.

\section{Charting the data}

An analytical method will be used to extract the background information and process oriented information of each included study. A data charting form will be developed and piloted. The following variables for which data will be sought in order to answer the question will be determined and will include author(s) names, date of publication, country of publication study title, journal full reference, aims or research question, participant characteristics, sampling method, study design, theoretical background, data collection, data analysis and most relevant findings conclusions and comments. The piloting forms will be done by independent reviewers in duplicate [28]. This will be done by ensuring that the reviewers independently conduct a trial data extraction and later discuss as a team to ensure that the approach is in line with the objective. To improve the applicability, consistency and quality of the chart, a continued review through an iterative process, which involves reading and re-reading the summaries, will be ensured. This process will entail continuous editing of the extraction form throughout the duration of the study.

\section{Collating, summarising, and reporting the results}

This study is aimed at mapping the existing evidence and to summarise the findings as presented across articles. The data from the data collection process will be quantitatively summarised using a simple numerical count and qualitatively drawn on the descriptiveanalytical method using thematic analysis and visual representations (including diagrams). The quantitative results will be presented in a table format, followed by a narrative section containing the main theories. A narrative account of all the data extracted from the studies that meet the inclusion criteria will be analysed using the thematic content analysis. Data relating to the beliefs and values of mothers or women, in regards to childbirth by skilled health care providers in Africa, will be coded using NVivo version 10 [29]. Emerging themes will be identified, coded, and categorised into major themes. The resulting themes will then be synthesised, summarised and discussed in relation to the objective of the study and its implications on ethical practice, policy and future research.

\section{Quality appraisal}

To determine the methodological quality of the studies to be included, we will use the mixed method appraisal tool (MMAT) version 2018 [30]. The MMAT tool will help us determine the quality of the studies by examining the aptness of the aim of the study and the adequacy 
of the methodology, study design, participant recruitment, data collection, data analysis, presentation of findings and authors' discussions and conclusions. A scoring matrix in the MMAT tool will be used to independently appraise the quality of the studies, and the results from the scoring will be used to determine the overall quality of the article.

\section{Discussion}

Efforts to identify drivers to non-use of SBA among women should consider different and additional dimensions including normative and ethical inquiry [31, 32]. Understanding the beliefs and values of mothers and others close to them, and how individual mothers are influenced by these beliefs and values, is critical to informing public health systems on the possible 'hidden' barriers and facilitators to utilisation of SBA in public health facilities. The findings from this review may inform public health and clinical practice and guide the design of strategies that respond to the beliefs and values of mothers, in efforts to achieve Universal Health Coverage for maternal health care interventions in developing countries.

Studies with no evidence of mothers' beliefs and values including perceptions and experiences surround child birth will be excluded because the focus of this review is on normative beliefs and values that shape care-seeking behaviours for SBA by mothers in Africa. This scoping review will be limited to articles presenting findings from Africa because of the similar health issues. Specifically, this study also focuses on when the views of women in Africa.

This scoping review may have limitations worth noting. Firstly, limitations in this scoping review may arise due to the focus on mapping the breadth of studies instead of the depth of information. However, the chosen methodology is appropriate in addressing the review questions. Secondly, whilst this study by drawing on mixed methods will draw on rich information for the objective, there may be difficulty arriving at overall meaningful summary measures across heterogeneous studies.

Nevertheless, this scoping review will be critical to uncover the breadth and extent of research that exists in field. The findings of the review may be of interest to researchers by highlighting research gaps that may need further investigation. Further, implementers and policymakers involved in interventions aimed at improving SBA may be informed by the evidence from the review. Finally, the review of findings from studies in Africa will be of interest to informing health systems on some demand side barriers and facilitators to utilisation of SBA in public health facilities. The findings of this scoping review study will be published in a peer-reviewed journal.
Abbreviations

SBA: Skilled birth attendance; PCC: Population, Concept, Context

\section{Supplementary Information}

The online version contains supplementary material available at https://doi. org/10.1186/s13643-021-01629-1.

Additional file 1. PRISMA-P (Preferred Reporting Items for Systematic review and Meta-Analysis Protocols) 2015 checklist.

Additional file 2. PUBMED Search Strategy Summary - Date; $12^{\mathrm{TH}}$ November 2020.

\section{Acknowledgements}

The authors are grateful for comments on the version from Joseph Ali and Mercy Monde.

\section{Authors' contributions}

CJ developed the first draft and subsequent revisions; $\mathrm{AH}$ helped edit and revise all versions. All authors approved the final version.

\section{Funding}

This work was produced under support of a grant from the National Institutes of Health, Fogarty International Center.

Availability of data and materials

Further details of the protocol are available from the corresponding author on reasonable request.

\section{Declarations}

Ethics approval and consent to participate

Not applicable.

Consent for publication

Not applicable.

\section{Competing interests}

The authors declare that they have no competing interests.

\section{Author details}

${ }^{1}$ School of Public Health, University of Zambia, Nationalist Road, Box 50110, Lusaka, Zambia. ${ }^{2}$ Berman Institute of Bioethics, Johns Hopkins University, Baltimore, MD, USA. ${ }^{3}$ Milken Institute School of Public Health, George Washington University, Washington, DC, USA.

Received: 31 May 2020 Accepted: 10 March 2021

Published online: 29 March 2021

\section{References}

1. Graham WJ, Bell JS, Bullough CH. Can skilled attendance at delivery reduce maternal mortality in developing countries? In: Safe motherhood strategies: a review of the evidence; 2001.

2. Friberg IK, Kinney MV, Lawn JE, Kerber KJ, Odubanjo MO, Bergh A-M, et al. Sub-Saharan Africa's mothers, newborns, and children: how many lives could be saved with targeted health interventions? PLoS Med. 2010;7(6): e1000295.

3. Ng'anjo SP, Fylkesnes K, Ruano AL, Moland KM. 'Born before arrival': user and provider perspectives on health facility childbirths in Kapiri Mposhi district, Zambia. BMC Pregnancy Childbirth. 2014;14:323.

4. Jacobs C, Moshabela M, Maswenyeho S, Lambo N, Michelo C. Predictors of antenatal care, skilled birth attendance, and postnatal care utilization among the remote and poorest rural communities of Zambia: a multilevel analysis. Front Public Health. 2017;5:11.

5. N'Gbichi C, Ziraba AK, Wambui DW, Bakibinga P, Kisiangani I, Njoroge P, et al. "If there are no female nurses to attend to me, I will just go and deliver at home": a qualitative study in Garissa, Kenya. BMC Pregnancy Childbirth. 2019;19(1):332. 
6. Adebowale SA, Akinyemi JO. Determinants of maternal utilization of health services and nutritional status in a rural community in South-West Nigeria. Afr J Reprod Health. 2016;20(2):72-85.

7. Kumbani L, Bjune G, Chirwa E, Odland JØ. Why some women fail to give birth at health facilities: a qualitative study of women's perceptions of perinatal care from rural Southern Malawi. Reprod Health. 2013;10(1):9.

8. Osubor K, Fatusi AO, Chiwuzie J. Maternal health-seeking behavior and associated factors in a rural Nigerian community. Matern Child Health J. 2006;10(2):159-69.

9. Maya ET, Adu-Bonsaffoh K, Dako-Gyeke P, Badzi C, Vogel JP, Bohren MA, et al. Women's perspectives of mistreatment during childbirth at health facilities in Ghana: findings from a qualitative study. Reprod Health Matters. 2018;26(53):70-87.

10. Ghaffar A, Pongponich S, Ghaffar N, Mehmood T. Factors associated with utilization of antenatal care services in Balochistan province of Pakistan: an analysis of the Multiple Indicator Cluster Survey (MICS) 2010. Pakistan J Med Sci. 2015;31(6):1447-52.

11. Subba D. Maternal complications and the utilisation of maternal health care services with special reference to West Bengal, India. Open J Obstet Gynecol. 2013;03(09):694-701

12. Choulagai B, Onta S, Subedi N, Mehata S, Bhandari GP, Poudyal A, Shrestha B, Mathai M, Petzold M, Krettek A. Barriers to using skilled birth attendants' services in mid-and far-western Nepal: a cross-sectional study. BMC Int Health Hum Rights. 2013;13(1):1-9.

13. Duong DV, Binns CW, Lee AH. Utilization of delivery services at the primary health care level in rural Vietnam. Soc Sci Med. 2004;59(12):2585-95.

14. Shaikh BT, Haran D, Hatcher J, lqbal Azam S. Studying health-seeking behaviours: collecting reliable data, conducting comprehensive analysis. J Biosoc Sci. 2008;40(01):53-68.

15. Shaikh BT, Hatcher J. Health seeking behaviour and health service utilization in Pakistan: challenging the policy makers. J Public Health. 2005;27(1):49-54.

16. Nyamongo I. Health care switching behaviour of malaria patients in a Kenyan rural community. Soc Sci Med. 2002;54(3):377-86.

17. Kyei-Nimakoh M, Carolan-Olah M, McCann TV. Access barriers to obstetric care at health facilities in sub-Saharan Africa-a systematic review. Syst Rev. 2017;6(1):110

18. Sialubanje C, Massar K, Hamer DH, Ruiter RA. Understanding the psychosocial and environmental factors and barriers affecting utilization of maternal healthcare services in Kalomo, Zambia: a qualitative study. Health Educ Res. 2014;29(3):521-32.

19. Stekelenburg J, Kyanamina S, Mukelabai M, Wolffers I, Van Roosmalen J. Waiting too long: low use of maternal health services in Kalabo, Zambia. Trop Med Int Health. 2004;9(3):390-8.

20. Gebremichael MW, Worku A, Medhanyie AA, Edin K, Berhane Y. Women suffer more from disrespectful and abusive care than from the labour pain itself: a qualitative study from Women's perspective. BMC Pregnancy Childbirth. 2018;18(1):392.

21. Lusambili AM, Naanyu V, Wade TJ, Mossman L, Mantel M, Pell R, Ngetich A, Mulama K, Nyaga L, Obure J, Temmerman M. Deliver on your own: disrespectful maternity care in rural Kenya. PloS one. 2020;15(1):e0214836.

22. Timmons M. Moral theory: an introduction: Rowman \& Littlefield Publishers; 2012.

23. Downe S, Finlayson K, Oladapo O, Bonet M, Gülmezoglu AM. What matters to women during childbirth: a systematic qualitative review. PLoS One. 2018;13(4):e0194906.

24. Tricco AC, Lillie E, Zarin W, O'Brien KK, Colquhoun H, Levac D, et al. PRISMA extension for scoping reviews (PRISMA-SCR): checklist and explanation. Ann Intern Med. 2018;169(7):467-73.

25. Arksey H, O'Malley L. Scoping studies: towards a methodological framework. Int J Soc Res Methodol. 2005;8(1):19-32.

26. Levac D, Colquhoun $\mathrm{H}$, O'Brien KK. Scoping studies: advancing the methodology. Implement Sci. 2010;5(1):69.

27. Peters M, Godfrey C, McInerney P, Soares C, Khalil H, Parker D. The Joanna Briggs Institute reviewers' manual 2015: methodology for JBI scoping reviews; 2015 .

28. Colquhoun HL, Levac D, O'Brien KK, Straus S, Tricco AC, Perrier L, et al. Scoping reviews: time for clarity in definition, methods, and reporting. J Clin Epidemiol. 2014;67(12):1291-4.

29. Castleberry A. NVivo 10 [software program]. Version 10. QSR International; 2012. Am J Pharm Educ. 2014;78:25. https://doi.org/10.5688/ajpe78125.
30. Hong QN, Fàbregues S, Bartlett G, Boardman F, Cargo M, Dagenais P, et al. The mixed methods appraisal tool (MMAT) version 2018 for information professionals and researchers. Educ Inf. 2018;34(4):285-91.

31. Prainsack B, Buyx A. Solidarity: reflections on an emerging concept in bioethics: Citeseer; 2011.

32. Dawson A, Verweij M. Solidarity: a moral concept in need of clarification: Oxford University Press; 2012

\section{Publisher's Note}

Springer Nature remains neutral with regard to jurisdictional claims in published maps and institutional affiliations.
Ready to submit your research? Choose BMC and benefit from:

- fast, convenient online submission

- thorough peer review by experienced researchers in your field

- rapid publication on acceptance

- support for research data, including large and complex data types

- gold Open Access which fosters wider collaboration and increased citations

- maximum visibility for your research: over $100 \mathrm{M}$ website views per year

At $\mathrm{BMC}$, research is always in progress.

Learn more biomedcentral.com/submissions 